\title{
A GAME-THEORETIC APPROACH FOR DETERMINING THE ECONOMIC MANUFACTURING QUANTITY AND MUTUAL INSPECTION POLICY UNDER A QUALITY CONTRACT
}

\author{
Chih-Hsiung Wang \\ National Pingtung Institute of Commerce
}

(Received November 23, 2007; Revised May 21, 2008)

\begin{abstract}
In this study, we deal with the optimal mutual inspection policies (OMIP) for a manufacturer and its downstream member (buyer) under a quality contract subject to the probability of non-conforming for each unit is transparent between the manufacturer and buyer. A game-theoretic framework is developed for both parties to determine their OMIP. Main difference between this study and related previous works is that in this research the full inspection policy will never be optimal for the manufacturer when a quality contract is considered. Based on this OMIP, we show how the OMIP is incorporated into the determination of optimal production lot size. To achieve an economical production under the defined contract, tradeoffs between manufacturing cost, inventory holding cost and quality related cost must be analyzed. Some properties for the optimal lot size are obtained. Numerical examples are performed to illustrate the optimal production lot size and OMIP.
\end{abstract}

Keywords: Game theory, inspection, EMQ

\section{Introduction}

It is known that product quality, innovative design, service difference and production cost are the major factors which determine whether a firm will win orders (e.g., see Martinich [7]). Today, quality issues are particular important, e.g., Olhager and Selldin [10] studied 128 different companies in Sweden and found that quality is the most important factor for firms in the selection of partners in supply chains, and that achieving cost minimization is the overall main objective in designing supply chains.

Various methodologies for controlling output quality have been studied in the past few decades, including the relationship between production lot size and the number of nonconforming units with warranty (e.g., see Yeh et al. [13]), or the effects of inspection schedules for imperfect production processes (e.g., see $[2,3,5,6,16])$, or the effects of inspecting units and the disposition of non-conforming units (e.g., see Raz et al. [12]), or the effects of improving process reliability by capital investment to reduce the likelihood of producing non-conforming units (e.g., see Porteus [11]). When product inspection, which is the subject of this paper, is performed to control product quality, the associated actions, such as disposition, salvage or rework are usually performed on the identified non-conforming units to prevent them from reaching customers. Without considering the nature of process manufacturing variability, Raz et al. [12] proposed an economic inspection/disposition model subject to off-line quality control. In their model, a solution procedure with dynamic programming is provided to obtain the optimal inspection policy. When the process possesses manufacturing variability, Wang [18] proposed an integrated product inspection/repair and 
production policy, where inspections are performed only at the tail segment of the production batch. In this policy, the solution procedure was proposed for the simultaneous determination of the optimal production lot size and inspection policy.

Although Raz et al. [12] tried to optimize the manufacturer's quality related control cost, and Wang [18] attempted to optimize the manufacturer's manufacturing cost, inventory holding cost and quality related control cost, these actions still may not satisfy the buyer's requirements for confidence in the output quality. Since a quality contract is an effective way of assuring buyers of output quality, Tapiero [15] developed a game theoretical model under a quality contract to establish the optimal mutual sampling policies. Recently, Narasimhan and Nair [9] found that the information shared between the supplier and buyer had a positive impact on strategic alliance formation. One of the benefits of sharing information across organizations is to reduce the "bullwhip effect" (e.g., see Simchi-Levi et al. [14]).

In this study, we used a game-theoretic model to study the effects of a quality contract on the OMIP, where the probability of non-conforming for each unit is transparent between the manufacturer $(M)$ and buyer $(B)$. Our objective is to determine the optimal production lot size for M, and the OMIP for both parties through the application of game theory.

The rest of this paper is organized as follows. In the next section, we define a quality contract that is used to guarantee product quality for the buyer. Under the quality contract, we solve the OMIP. Then, the optimal production lot size is further determined. Properties for the optimal production lot sizes are investigated. Numerical examples are given to illustrate the OMIP and the optimal production lot size for the manufacturer. Finally, some possible extensions of the model are brought up.

\section{Quality Contract and Its Optimal Policy}

We consider a process for producing a single item and discrete unit, where the process has two states, in-control and out-of-control. Product quality is classified as conforming or nonconforming. When the process is in the in-control (or the out-of-control) state, there is a probability of $\theta_{1}$ (or $\theta_{2}$ ) that the produced unit will be non-conforming and $1-\theta_{1}$ (or $1-\theta_{2}$ ) that it will be conforming, where $0 \leq \theta_{1}<\theta_{2} \leq 1$. At the beginning of each production lot, the process is setup including maintenance with joint cost $K$ so that it is in the in-control state. Let random variable $Z$ represent the number of produced units when the process transitions to the out-of-control state since the last setup. As in Wang and Sheu [17], let $\bar{P}_{j}=P(Z>j)$ represent the reliability that the first $j$ units are produced in the in-control state since the last setup. Once the process shifts to the out-of-control state, it will not be restored until the beginning of the next production run. The probability that the $j$ th unit will be a non-conforming is readily obtained as

$$
q_{j}=\theta_{2}-\left(\theta_{2}-\theta_{1}\right) \bar{P}_{j}
$$

which is increasing in $j$.

To assure the buyers' right of quality when a non-conforming unit is received, a quality contract is considered. There are several types of quality contracts in the real world, e.g., Jones [4] studied the forms of contracts used in the UK and Republic of Ireland. The classic style is the "fixed price" contract, where all non-conforming costs are entirely attributed to the supplier. In this case, suppliers may hesitate to go forward with the contract since there may be a problem if the number of non-conforming units is seriously underestimated. Furthermore, the contract that entails an agreed proportion of the cost of the non-conforming items that is defrayed by the buyers is called the "cost-plus" contract; however, the costs 
Table 1: (a). The random payoff matrix of the $j$ th unit for $M(B)$

\begin{tabular}{lll} 
& B: Inspection & B: No inspection \\
\hline M: Inspection & $\pi-c_{M}-c_{I}$ w.p. $1-q_{j}$ & $\pi-c_{M}-c_{I}$ w.p. $1-q_{j}$ \\
& $\left(\phi-\pi-c_{B}\right)$ & $(\phi-\pi)$ \\
& $\pi-c_{M}-c_{I}-c_{R}$ w.p. $q_{j}$ & $\pi-c_{M}-c_{I}-c_{R}$ w.p. $q_{j}$ \\
& $\left(\phi-\pi-c_{B}\right)$ & $(\phi-\pi)$ \\
\hline M: No inspection & $\pi-c_{M}$ w.p. $1-q_{j}$ & $\pi-c_{M}$ w.p. $1-q_{j}$ \\
& $\left(\phi-\pi-c_{B}\right)$ & $(\phi-\pi)$ \\
& $\pi-c_{M}-T$ w.p. $q_{j}$ & $\pi-c_{M}$ w.p. $q_{j}$ \\
& $\left(T-\pi-c_{B}\right)$ & $\left(\phi-\pi-c_{W}\right)$ \\
\hline
\end{tabular}

Table 1: (b). The expected random payoff matrix of the $j$ th unit for $\mathrm{M}(\mathrm{B})$

\begin{tabular}{lll} 
& B: Inspection & B: No inspection \\
\hline M: Inspection & $u_{j}^{11}=\pi-c_{M}-c_{I}-q_{j} c_{R}$ & $u_{j}^{12}=u_{j}^{11}$ \\
& $\left(v_{j}^{11}=\phi-\pi-c_{B}\right)$ & $\left(v_{j}^{12}=\phi-\pi\right)$ \\
\hline M: No inspection & $u_{j}^{21}=\pi-c_{M}-q_{j} T$ & $u_{j}^{22}=\pi-c_{M}$ \\
& $\left(v_{j}^{21}=q_{j} T+\left(1-q_{j}\right) \phi-\pi-c_{B}\right)$ & $\left(v_{j}^{22}=\phi-\pi-q_{j} c_{W}\right)$ \\
\hline
\end{tabular}

may be exaggerated by suppliers. In our study, a penal contract subject to the probability of non-conforming for each unit which was transparent between $M$ and $B$ was introduced so that the risks for both parties mentioned in the above quality contracts were diminished. The quality contract is defined as follows.

I. Suppose that $\mathrm{M}$ inspects the $j$ th unit in a batch with a cost $c_{I}>0$. If it is non-conforming, then it is repaired to be conforming, with repair cost $c_{R}$.

II. When $\mathrm{M}$ does not inspect the $j$ th unit, which is a non-conforming unit, the cost incurred on $\mathrm{M}$ for the $j$ th unit depends on the behavior of $\mathrm{B}$ upon receiving it:

(i) if $\mathrm{B}$ does not inspect it, then $\mathrm{B}$ will incur a warranty cost $c_{W}$ when this unit reaches the market. In this case, $M$ does not pay any penalty to $B$.

(ii) If $\mathrm{B}$ inspects it with inspection $\operatorname{cost} c_{B}$ and finds it to be non-conforming, $\mathrm{M}$ must pay a penalty $T$ to $\mathrm{B}$. It is reasonable to assume that $T>\pi+c_{B}$, where $\pi$ is the selling price per unit for $B$.

III. $\mathrm{M}$ must share its quality information $q_{j}$ with $\mathrm{B}$.

According to the contract mentioned above, a random payoff matrix for the $j$ th unit is given in Table 1(a), where $\phi$ is the selling price per unit for the market (end customer) and $c_{M}$ is the manufacturing cost per unit for M. From Table 1(a), the value of the expected random payoff matrix is obtained as shown in Table 1(b). Denote $x_{j}$ and $y_{j}$ as the probability that the $j$ th unit will be inspected by $\mathrm{M}$ and $\mathrm{B}$, respectively. From Table $1(\mathrm{~b})$, the expected payoff of the $j$ th unit for $\mathrm{M}$ and $\mathrm{B}$ under their inspection policies, $\left(x_{j}, y_{j}\right)$, can be obtained as follows:

$$
U_{j}\left(x_{j}, y_{j} ; q_{j}\right)=\left\{T q_{j} y_{j}-\left(c_{I}+c_{R} q_{j}\right)\right\} x_{j}+\pi-c_{M}-T y_{j} q_{j},
$$

$V_{j}\left(x_{j}, y_{j} ; q_{j}\right)=\left\{\left(\phi-c_{W}-T\right)\left(x_{j}-1\right) q_{j}-c_{B}\right\} y_{j}+\phi-\pi+c_{W}\left(x_{j}-1\right) q_{j}$, respectively.

Now we proceed to obtain the OMIP. When M incurs $c_{I}$ to inspect a unit, the maximum 
return, $T-c_{R}$, occurs when the inspected unit is non-conforming and B inspects it. Denote $H=c_{I} /\left(T-c_{R}\right)>0$ as the cost ratio for M, i.e., the "inspection" to "the possible maximum return." On the other hand, when B incurs $c_{B}$ to inspect a received unit, the maximum return, $T-\left(\phi-c_{W}\right)$, occurs when the inspected unit is non-conforming and $\mathrm{M}$ does not inspect it. Denote $G=c_{B} /\left(T+c_{W}-\phi\right)>0$ as the cost ratio for B, i.e., "inspection" to "the possible maximum return." Intuitively, if $H$ (or $G$ ) is small, then M (or B) is prone to inspect the unit. However, the OMIP should consider the non-conforming probability for a unit and the inspection behaviors of the other side.

The discussed game for the OMIP is known as the "Stackelberg leadership model" [13] where $\mathrm{M}$ is the "leader" and $\mathrm{B}$ is the "follower", i.e., $\mathrm{M}$ inspects unit $j$ first and then $\mathrm{B}$ does. In addition, when a mutual inspection probability exists for unit $j$, the solution $\left(x_{j}^{*}, y_{j}^{*}\right)$ for the OMIP can be found by solving $\partial \sum_{j=1}^{n} U_{j}\left(x_{j}, y_{j} ; q_{j}\right) / \partial x_{j}=0$ and $\partial \sum_{j=1}^{n} V_{j}\left(x_{j}, y_{j} ; q_{j}\right) / \partial y_{j}=0$, simultaneously, which is also known as the subgame perfect Nash equilibrium (e.g., see Gibbons [1]). The OMIP for the $j$ th unit is given in the following property.

Property 1. Four mutually exclusive and exhaustively collected sets are defined as: $J_{A}=\left\{j \mid q_{j} \leq H, q_{j} \leq G\right\}, J_{B}=\left\{j \mid q_{j} \leq H, q_{j}>G\right\}, J_{C}=\left\{j \mid q_{j}>H, q_{j} \leq G\right\}$ and $J_{D}=\left\{j \mid q_{j}>H, q_{j}>G\right\}$. The OMIP for the $j$ th unit can be obtained as follows. When $j \in J_{A}, j \in J_{B}, j \in J_{C}$ or $j \in J_{D}$, we have $\left(x_{j}^{*}, y_{j}^{*}\right)=(0,0),(0,1),(0,0)$ or $\left(1-G / q_{j},\left(c_{I}+q_{j} c_{R}\right) /\left(q_{j} T\right)\right)$, respectively.

Property 1 reveals that when $j \in J_{A}$ (the unit's quality is possibly excellent, or equivalently, the inspection costs for $\mathrm{M}$ and $\mathrm{B}$ are too high), neither $\mathrm{M}$ nor $\mathrm{B}$ will perform an inspection. When $j \in J_{B}$, it implies that $\mathrm{M}$ does not inspect unit $j$ because of its high inspection cost while $\mathrm{B}$ will inspect unit $j$ since the inspection cost is relatively low. When $j \in J_{C}$, it indicates that B will not perform an inspection for unit because of the high inspection cost, and $\mathrm{M}$ will not inspect unit $j$ either, even though the inspection costs for $\mathrm{M}$ are relatively low. This is because when $\mathrm{M}$ inspects unit $j$, it merely increases the inspection costs and there is no possible return from the inspection since B never inspects unit $j$. Finally, when $j \in J_{D}$, a mutual inspection probability exists for unit $j$ since the defined quality game follows the "Stackelberg leadership model" [13], and the OMIP can be found by solving $\partial \sum_{j=1}^{n} U_{j}\left(x_{j}, y_{j} ; q_{j}\right) / \partial x_{j}=0$ and $\partial \sum_{j=1}^{n} V_{j}\left(x_{j}, y_{j} ; q_{j}\right) / \partial y_{j}=Q$, simultaneously. The solution $\left(x_{j}^{*}, y_{j}^{*}\right)=\left(1-G / q_{j},\left(c_{I}+q_{j} c_{R}\right) /\left(q_{j} T\right)\right)$ implicates that nobody has incentive to unilaterally change his/her inspection probability on unit $j$. In this case, $\mathrm{M}$ will focus on the units produced in the tail segment of the batch since they are prone to be non-conforming, whereas, B will perform an inspection that focuses on the units produced in the initial segment since $M$ is less likely to inspect them.

From Property 1, we know that the inspection probability for the $j$ th unit is increasing in $j$ for $\mathrm{M}$, and a full inspection policy will never be optimal. Next, a simple decision rule for the OMIP is obtained from Property 1 as follows.

\section{Corollary 1.}

Case 1. $G<H$ : When $q_{j} \leq G, G<q_{j} \leq H$ and $H<q_{j} \leq 1$, we have the OMIP $\left(x_{j}^{*}, y_{j}^{*}\right)$ are $(0,0),(0,1)$ and $\left(1-G / q_{j},\left(c_{I}+q_{j} c_{R}\right) /\left(q_{j} T\right)\right)$, respectively. 
Case 2. $G \geq H$ : When $q_{j} \leq G$ and $G<q_{j} \leq 1$, we have the OMIP $\left(x_{j}^{*}, y_{j}^{*}\right)$ are $(0,0)$ and $\left(1-G / q_{j},\left(c_{I}+q_{j} c_{R}\right) /\left(q_{j} T\right)\right)$, respectively.

In Corollary 1, when the inspection cost for $\mathrm{M}$ and $\mathrm{B}$ are equal (e.g., $\mathrm{M}$ and $\mathrm{B}$ share the same inspection equipments), if M's repair cost for a non-conforming unit is greater than the "gross revenue" of $\mathrm{B}$ when a non-conforming unit is sold in the market (i.e., $c_{R}>\phi-c_{W}$ ), then we have $G<H$; otherwise, if $c_{R} \leq \phi-c_{W}$, then we have $G \geq H$. Note that if B's net revenue $\phi-\pi-c_{W}$ is not negative and the reworked cost $c_{R}$ is less than the selling price $\pi$, then $c_{R} \leq \phi-c_{W}$ holds.

Utilizing $\left(x_{j}^{*}, y_{j}^{*}\right)$ obtained in Corollary 1 , for a given lot of size $n$, the expected payoff per unit of $\mathrm{M}$ is $\sum_{j=1}^{n} U_{j}^{*} / n$, which reflects cost and is negative, where $U_{j}^{*} \equiv U\left(x_{j}^{*}, y_{j}^{*} ; q_{j}\right)$. Combining the setup cost and inventory holding cost, the expected total cost per unit for $\mathrm{M}$ under the OMIP for the given quality contract is given as

$$
\operatorname{ETC}\left(n ;\left\{q_{j}\right\}\right)=K / n+h n /(2 d)-\sum_{j=1}^{n} U_{j}^{*} / n,
$$

where $h$ is the inventory carrying cost per unit product per unit time and the expected inventory carrying cost is derived under the assumption that the production rate is relatively larger than the demand rate, $d$. Now, we seek to find the optimal value $n^{*}$ by minimizing the cost function given in Equation (2). The necessary condition for $n^{*}$ is to satisfy

$$
\operatorname{ETC}\left(n ;\left\{q_{j}\right\}\right)<\operatorname{ETC}\left(n-1 ;\left\{q_{j}\right\}\right) \text { and } \operatorname{ETC}\left(n ;\left\{q_{j}\right\}\right) \leq \operatorname{ETC}\left(n+1 ;\left\{q_{j}\right\}\right) .
$$

Equation (3) can be rearranged as

$$
\begin{gathered}
\Theta\left(n-1 ;\left\{q_{j}\right\}\right)<K \text { and } \Theta\left(n ;\left\{q_{j}\right\}\right) \geq K \text { where } \\
\Theta\left(n ;\left\{q_{j}\right\}\right)=\left\{\begin{array}{l}
h n(n+1) /(2 d)+\sum_{j=1}^{n} U_{j}^{*}-n U_{n+1}^{*}, \text { for } n=1,2, \cdots, \\
0, \text { for } n=0 .
\end{array}\right.
\end{gathered}
$$

The following property explicates the behavior $U_{j}^{*}$, and will be useful in determining $n^{*}$ for the later analysis.

Property 2. If $G<H$, we have $U_{j}^{*}=U_{j+1}^{*}$, for $j \leq\left|J_{A}\right|-1$, and $U_{j}^{*}>U_{j+1}^{*}$, for $\left|J_{A}\right| \leq j<\infty$. On the other hand, if $G \geq H$, we have $U_{j}^{*}=U_{j+1}^{*}$, for $j \leq\left|J_{A}\right|+\left|J_{C}\right|-1$, and $U_{j}^{*}>U_{j+1}^{*}$, for $\left|J_{A}\right|+\left|J_{C}\right| \leq j<\infty$.

In Property 2 we saw that M's expected payoff for the $j$ th unit is decreasing in $j$ under the OMIP. Based on this property, we further develop the following property for $n^{*}$.

Property 3. Let $Q_{\mathrm{E}}=\sqrt{2 K d / h}$. The optimal production lot size $\left(n^{*}\right)$ can be uniquely determined by Equations (4)-(5), and $n^{*} \in\left[0, Q_{\mathrm{E}}\right]$ if $\left|J_{A}\right|<Q_{\mathrm{E}}$, or $n^{*}=Q_{\mathrm{E}}$ if $\left|J_{A}\right| \geq Q_{\mathrm{E}}$, for $G<H$. On the other hand, when $G \geq H$, if $\left|J_{A}\right|+\left|J_{C}\right|<Q_{\mathrm{E}}$, then we have $n^{*} \in\left[0, Q_{\mathrm{E}}\right]$, or if $\left|J_{A}\right|+\left|J_{C}\right| \geq Q_{\mathrm{E}}$, then $n^{*}=Q_{\mathrm{E}}$.

Property 3 implicates that when the process reliability is good, no inspection is needed and the optimal production lot size is equal to the classical economic order quantity (EOQ), $Q_{\mathrm{E}}$.

We summarize the above findings in the following algorithm for $n^{*}, x_{j}^{*}$ and $y_{j}^{*}$ (note that all the values with index $j$ in Steps 1-3 are calculated from $j=1$ to $j=Q_{\mathrm{E}}+1$ since the term $U_{Q_{E}+1}^{*}$ in Step 3 maybe used for obtaining $n^{*}$ ): 
Step 1. Input the model parameters and compute $q_{j}=\theta_{2}-\left(\theta_{2}-\theta_{1}\right) \bar{P}_{j}, H=c_{I} /\left(T-c_{R}\right)$ and $G=c_{B} /\left(T+c_{W}-\phi\right)$.

Step 2. If $G<H$, then $\left(x_{j}^{*}, y_{j}^{*}\right)$ is $(0,0),(0,1)$ or $\left(1-G / q_{j},\left(c_{I}+q_{j} c_{R}\right) /\left(q_{j} T\right)\right)$ for $q_{j} \leq$ $G, G<q_{j} \leq H$ or $H<q_{j} \leq 1$, respectively. If $G \geq H$, then $\left(x_{j}^{*}, y_{j}^{*}\right)$ is $(0,0)$ or $\left(1-G / q_{j},\left(c_{I}+q_{j} c_{R}\right) /\left(q_{j} T\right)\right)$ for $q_{j} \leq G$ or $G<q_{j} \leq 1$, respectively.

Step 3. If $\left|J_{A}\right| \geq Q_{\mathrm{E}}$ for $G<H$, or if $\left|J_{A}\right|+\left|J_{C}\right| \geq Q_{\mathrm{E}}$ for $G \geq H$, then $n^{*}=Q_{\mathrm{E}}$. Otherwise, $n^{*}$ can be found at the smallest $n$ that satisfies $h n(n+1) / 2+\sum_{j=1}^{n} U_{j}^{*}-n U_{n+1}^{*} \geq K$. Stop.

A binary search algorithm can be used in Step 3 with the maximum number of iterations $Q_{\mathrm{E}} \log \left(Q_{\mathrm{E}}\right)$ for $n^{*}$.

Remark 1. In Porteus's [11] model we know that the manufacturer performed full inspection and all non-conforming units are reworked with a certain cost, but the inspection cost is negligible. Setting $\theta_{1}=0, \theta_{2}=1, c_{M}=0, \pi=0, \phi=0, c_{W}=0, x_{j}=1$, $y_{j}=0$ and $\bar{P}_{j}=p^{j}$ in Equation (2) gives

$$
\operatorname{ETC}\left(n ;\left\{q^{j}\right\}\right)=\frac{K}{n}+\frac{h n}{2 d}-c_{R}\left(1-\frac{p\left(1-p^{n}\right)}{n(1-p)}\right)
$$

which is exactly the result obtained by Porteus [11]. In this case, it is easy to verify that the condition given in Property 2 holds since $U_{j}^{*}=u_{j}^{12}=-q_{j} c_{R}>U_{j+1}^{*}$, for all $j$.

Subsequently, we can apply Property 3 to confirm that Porteus's [11] optimal lot size is unique (also see Wang and Sheu [17]).

Remark 2. The main difference between our study and related previous studies (e.g., Porteus [11]) is that in our research the full inspection policy can never be optimal for the manufacturer when a quality contract is considered.

Remark 3. Based on the OMIP, the optimal production lot size is shown to be less or equal to the classic EOQ. This agrees with the results found by Porteus [11], but the property may not hold for some existing production lot size models, where the restoration cost (e.g., see Yeh et al. [19]) and/or the process inspection schedule is an additional consideration (e.g., see Makis [6]). Thus, a larger production lot size than EOQ is needed so that these costs can be balanced.

\section{Numerical Examples}

To illustrate the optimal solution, $\left(x_{j}^{*}, y_{j}^{*}\right)$ and $n^{*}$, for the discussed problem, we carried out a numerical example with the following nominal data: $\theta_{1}=0.05, \theta_{2}=0.95, d=250, \pi=3$, $\phi=4, c_{M}=0.5, c_{B}=0.4, h=1.5, c_{I}=0.3, c_{R}=0.5, c_{W}=1.5, T=4$ and $K=10$. We used a discrete Weibull distribution to represent the process life time distribution, which is one of most widely used in reliability modeling. More precisely, we set $\bar{P}_{j}=p^{j^{\alpha}}$ (see Nakagawa and Osaki [8]). Various combinations of parameter values $\alpha$ and $p$ are selected to investigate the changes in $n^{*}, x_{j}^{*}$ and $y_{j}^{*}$, where $p$ takes on the values $0.9,0.95$, and 0.97 , and $\alpha$ takes on the values on $0.7,1.0$, and 1.3 that represent the decreasing, constant and increasing failure rates, respectively [8]. The results are summarized in Table 2, where $n^{*}$ will be located in the interval $\left[n_{1}, n_{2}\right]$, namely an effective production range, and all of the production lot size belongs within the range which creates a negative cost that reflects a positive payoff. This is because the cost function given in Equation (2) is convex from the proof of Property 3. 
Table 2: The optimal production lot size for different process reliabilities

\begin{tabular}{rrrrrrrrrr}
$p$ & 0.9 & 0.95 & 0.97 & 0.9 & 0.95 & 0.97 & 0.9 & 0.95 & 0.97 \\
$\alpha$ & 1.3 & 1.3 & 1.3 & 1 & 1 & 1 & 0.7 & 0.7 & 0.7 \\
$n^{*}$ & 49 & 41 & 33 & 43 & 34 & 31 & 40 & 33 & 23 \\
$E T C^{*}$ & -1.433 & -1.479 & -1.552 & -1.474 & -1.593 & -1.708 & -1.593 & -1.773 & -1.996 \\
$n_{1}$ & 5 & 5 & 5 & 5 & 5 & 5 & 5 & 5 & 5 \\
$n_{2}$ & 570 & 572 & 573 & 571 & 575 & 579 & 577 & 593 & 613 \\
\hline
\end{tabular}

From Table 2, we know that for a fixed $\alpha$ (or $p$ ), the process probability is increasing (or decreasing) in $p$ (or $\alpha$ ). We observe the effects of process reliability on the optimal production lot size, expected total cost per unit and OMIP as follows.

(1) For a fixed $\alpha$ (or $p$ ), the number of uninspected units is increasing (or decreasing) in $p$ ( or $\alpha$ ) for both parties. This is because the initial segment in a batch is more likely to be conforming when the process possesses higher reliability.

(2) When a process has a higher reliability, it lowers the cost per unit, but allows a smaller lot size to compensate for the setup cost.

(3) The plot of OMIP and cost function for $p=0.97$ and $\alpha=1.3$ are given in Figures 1 and 2, respectively. The illustrated numerical example in this section corresponds to the Case 2 of Corollary 1 since $G>H$. It is easy to see that Figure 1 corroborates the results of the OMIP stated in Case 2 of Corollary 1, e.g., M will pay more attention to the tail segment of the batch. Furthermore, under the OMIP, Figure 2 reveals the fact that the total cost per unit is a convex function of the production lot size as shown in the proof of the Property 3.

(4) Finally, $n_{1}$ and $n_{2}$ are insensitive to changes in process reliability.

\section{Conclusions}

This paper investigates the effect of a quality contract between Manufacturer $(M)$ and Buyer (B) in a supply chain on the optimal production lot size and mutual inspection policy. Main difference between this study and related previous works (e.g., see Porteus [11]) is that in this research the full inspection policy will never be optimal for the manufacturer when a quality contract is considered. In addition, an algorithm with the maximum number of iterations $Q_{\mathrm{E}} \log \left(Q_{\mathrm{E}}\right)$ is proposed based on three proofed properties to determine the optimal inspections policy and the optimal production lot size. Extensions to this work could consider the possible existence of inspection errors, and constraints on the average probability for a non-conforming unit that will reach the market. It would also be interesting to study how capital investment might enhance the process reliability so that inspection does not need to be performed by either party. This will prevent inspection errors resulting from tedious inspections, and save inspection time so that the unit can reach the market sooner. It is also important to study the coordination of manufacturing/ordering quantity and inspection policies between the manufacturer and buyer.

\section{Acknowledgments}

The author would like to express his appreciation to the two anonymous referees for their very helpful comments and suggestions. All their suggestions were incorporated directly 


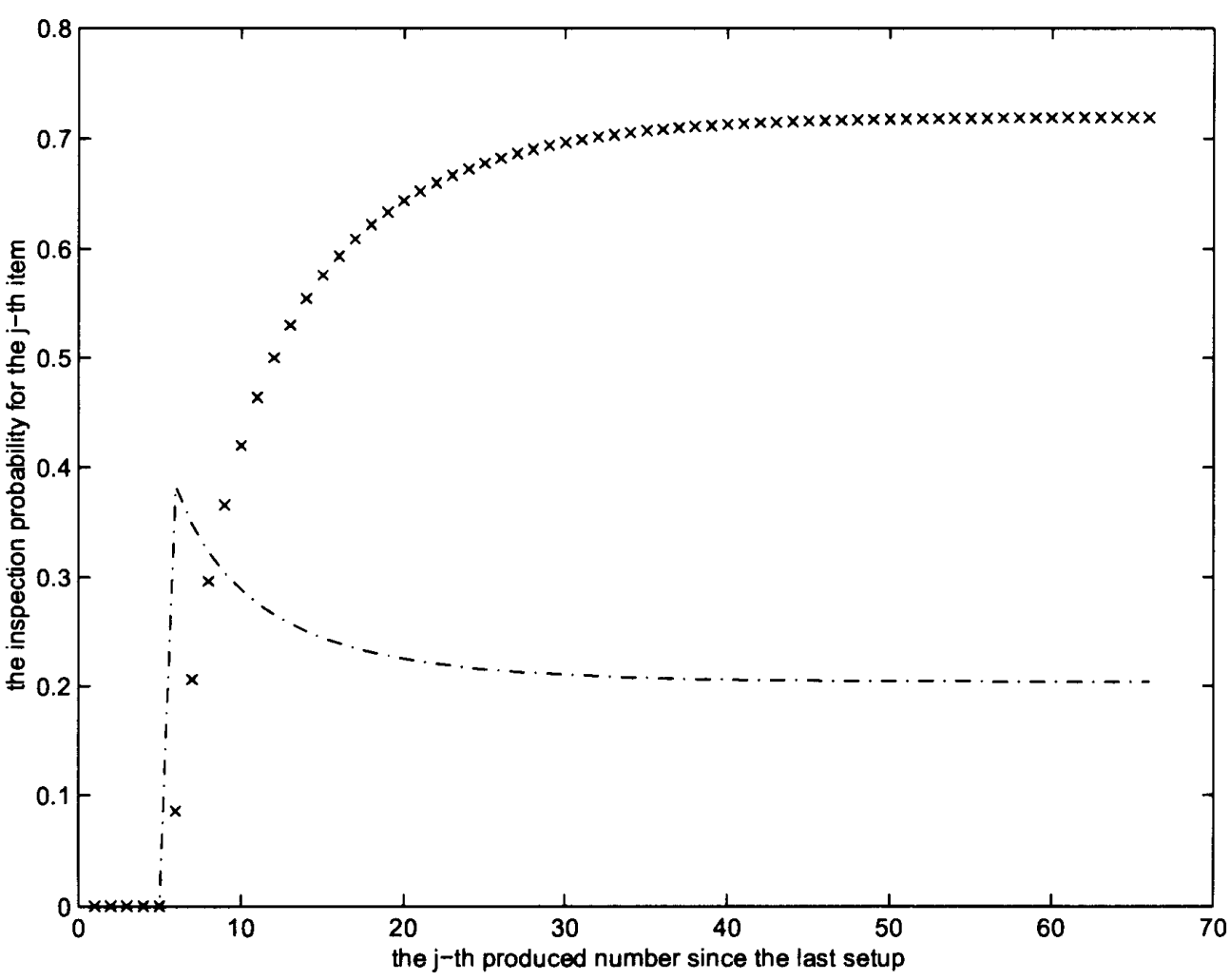

Figure 1: The plot of inspection probability for the $j$ th unit since the last setup, where the signs of " $x$ " and "-." represent the manufacturer's and buyer's inspection policy, respectively $(p=0.7$ and $\alpha=1.3)$

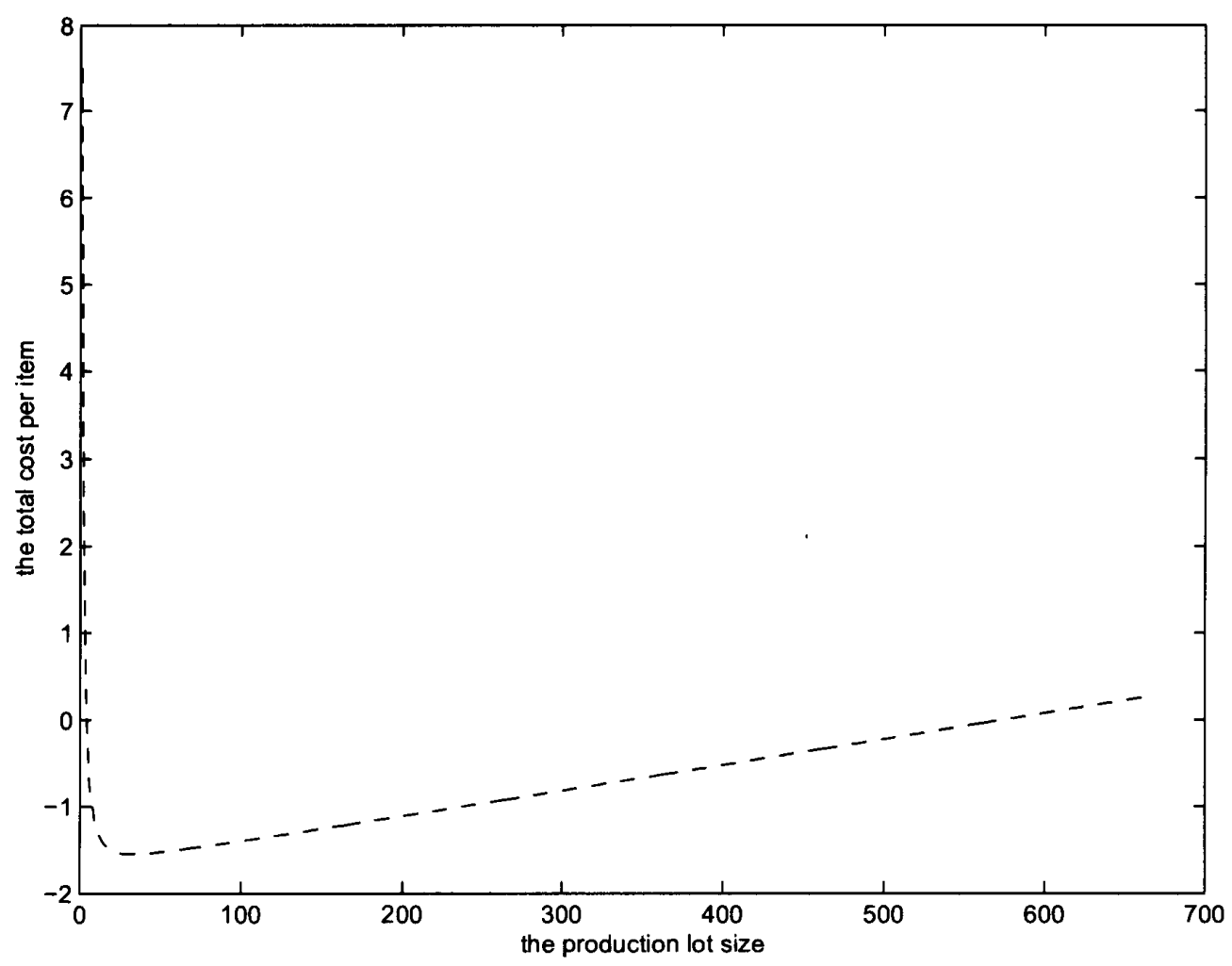

Figure 2: The cost function per unit for $p=0.97$ and $\alpha=1.3$ 
into the text. This research was supported by the grants (NSC-91-2213-E-251-005- and NSC-92-2213-E-251-003-) from the National Science Council, Taiwan.

\section{Appendix}

\section{Proof of Property 1.}

When $c_{I} \geq q_{j}\left(T-c_{R}\right)$, or equivalently, $q_{j} \leq H$, we have $u_{j}^{22} \geq u_{j}^{12}$ and $u_{j}^{21} \geq u_{j}^{11}$. This implies that $x_{j}^{*}=0$. Furthermore, when $c_{I} \geq q_{j}\left(T-c_{R}\right)$, we have $y_{j}^{*}=0$ if $c_{B} \geq q_{j}\left(T+c_{W}-\phi\right)$, or equivalently, $q_{j} \leq G$, and $y_{j}^{*}=1$ if $c_{B}<q_{j}\left(T+c_{W}-\phi\right)$. These result in (i) and (ii). Next, when $c_{B} \geq q_{j}\left(T+c_{W}-\phi\right)$, we know that $v_{j}^{22} \geq v_{j}^{21}$ and $v_{j}^{12} \geq v_{j}^{11}$. This implies that $y_{j}^{*}=0$. Furthermore, when $c_{B} \geq q_{j}\left(T+c_{W}-\phi\right)$, we have $x_{j}^{*}=0$ for $c_{I} \geq q_{j}\left(T-c_{R}\right)$ or $c_{I}<q_{j}\left(T-c_{R}\right)$. This shows (i) and (iii). In addition, when $c_{I}<q_{j}\left(\bar{T}-c_{R}\right)$ and $c_{B}<q_{j}\left(T+c_{W}-\phi\right), x_{j}^{*}$ and $y_{j}^{*}$ can be found by solving $\partial \sum_{j=1}^{n} U_{j}\left(x_{j}, y_{j} ; q_{j}\right) / \partial x_{j}=0$ and $\partial \sum_{j=1}^{n} V_{j}\left(x_{j}, y_{j} ; q_{j}\right) / \partial y_{j}=0$, simultaneously. $\quad$ Q.E.D.

\section{Proof of Property 2.}

We first consider the case that $G<H$. Recall that $q_{j}$ is increasing in $j$. When $q_{j} \leq G$, we have $j \in J_{A}$. This implies that $U_{j}^{*}=u_{j}^{22}$, for $j=1,2,3, \cdots,\left|J_{A}\right|$. Further, when $G<q_{j} \leq H$, we have $j \in J_{B}$. Using the monotonic property of $q_{j}$, we have $U_{j}^{*}=u_{j}^{21}<u_{j}^{22}$, for $j=\left|J_{A}\right|+1,\left|J_{A}\right|+2, \cdots,\left|J_{A}\right|+\left|J_{B}\right|$. When $H<q_{j}$, we have $j \in J_{D}$. Again, using the monotonic increasing property of $q_{j}$, from Equation (1) we have $U_{j}^{*}=u_{j}^{11}<u_{j}^{21}$ since $H<q_{j}$, for $j=\left|J_{A}\right|+\left|J_{B}\right|+1,\left|J_{A}\right|+\left|J_{B}\right|+2, \cdots$ In addition, it is easy to verify that $u_{j}^{22}=u_{j+1}^{22}$, $u_{j}^{21}>u_{j+1}^{21}$ and $u_{j}^{11}>u_{j+1}^{11}$, for all $j$. These imply that $U_{j}^{*}=U_{j+1}^{*}, j=1,2,3, \cdots,\left|J_{A}\right|-1$, and $U_{j}^{*}>U_{j+1}^{*}, j=\left|J_{A}\right|,\left|J_{A}\right|+1, \cdots$.

Q.E.D.

Next, we show the case that $G \geq H$ as follows. When $q_{j} \leq H \leq G$, we have $j \in J_{A}$. This implies that $U_{j}^{*}=u_{j}^{22}$, for $j \leq\left|J_{A}\right|$. When $H<q_{j} \leq G$, we have $j \in J_{C}$. This implies that $U_{j}^{*}=u_{j}^{22}$, for $j=\left|J_{A}\right|+1,\left|J_{A}\right|+2, \cdots,\left|J_{A}\right|+\left|J_{C}\right|$. Finally, when $H \leq G<q_{j}$, we have $j \in J_{D}$. This implies that $U_{j}^{*}=u_{j}^{11}$, for $j \geq\left|J_{A}\right|+\left|J_{C}\right|+1$. Besides, we can verify that $u_{j}^{22}=u_{j+1}^{22}, u_{j}^{22}>u_{j}^{11}$ and $u_{j}^{11}>u_{j+1}^{11}$, for all $j$. These conclude that $U_{j}^{*}=U_{j+1}^{*}$, $j=1,2,3, \cdots,\left|J_{A}\right|+\left|J_{C}\right|-1$, and $U_{j}^{*}>U_{j+1}^{*}, j \geq\left|J_{A}\right|+\left|J_{C}\right|$.

Q.E.D.

\section{Proof of Property 3.}

Now we show the case that $G<H$. First note that $\Theta\left(0 ;\left\{\bar{P}_{j}\right\}\right)=0$ and $\Theta\left(Q_{\mathrm{E}} ;\left\{\bar{P}_{j}\right\}\right) \geq$ $K$. This implies that there is a local minimum solution that exists in the finite interval $\left[0, Q_{\mathrm{E}}\right]$. Furthermore, from Properties 2 it is easy to see that $\Theta\left(n ;\left\{\bar{P}_{j}\right\}\right)$ is increasing in $n$, since $\Theta\left(n+1 ;\left\{\bar{P}_{j}\right\}\right)-\Theta\left(n ;\left\{\bar{P}_{j}\right\}\right)=(n+1)\left(h / d+U_{n+1}^{*}-U_{n+2}^{*}\right)>0$, for all $n \geq 0$. If $\left|J_{A}\right| \geq Q_{\mathrm{E}}$, then from Equation (5), we have $\Theta\left(n ;\left\{\vec{P}_{j}\right\}\right)<K$ for $n=1,2, \cdots,\left|J_{A}\right|$ since $U_{n}^{*}=0$ for $n \leq\left|J_{A}\right|$. This implies that $n^{*}=Q_{\mathrm{E}}$ when $\left|J_{A}\right| \geq Q_{\mathrm{E}}$. However, if $\left|J_{A}\right|<Q_{\mathrm{E}}$ then we only know that $n^{*} \leq Q_{\mathrm{E}}$. In the similar manners, we can show the case of $G \geq H$.

Q.E.D.

\section{References}

[1] R. Gibbons: Game theory for applied economists (Princeton University Press, 1992).

[2] B.C. Giri and T. Dohi: Optimal inspection schedule in an imperfect EMQ model with free repair warranty policy. Journal of the Operations Research Society of Japan, 49 (2006), 222-237. 
[3] B.C. Giri and T. Dohi: Inspection scheduling for imperfect production processes under free repair warranty contract. European Journal of Operational Research, 183 (2007), $238-252$.

[4] G.L. Jones: The impact of regulatory legislation on contractual costs risk and the form of contracts used in the UK and Republic of Ireland. European Journal of Purchasing \& Supply Management, 3-3 (1997), 137-145.

[5] J.S. Lee and K.S. Park: Joint Determination of Production Cycle and Inspection Intervals in a Deteriorating Production System. The Journal of the Operational Research Society, 42-9 (1991), 775-783.

[6] V. Makis: Optimal lot sizing and inspection policy for an EMQ model with imperfect inspections. Naval Research Logistics, 45 (1998), 165-186.

[7] J.S. Martinich: Production and operations management: An applied analytical approach (John Wiley and Sons, 1996).

[8] T. Nakagawa and S. Osaki: The discrete Weibull distribution. IEEE Transactions on Reliability, R-24-5 (1975), 300-301.

[9] R. Narasimhan and A. Nair: The antecedent role of quality, information sharing and supply chain proximity on strategic alliance formation and performance. International Journal of Production Economics, 96 (2005), 301-313.

[10] J. Olhager and E. Selldin: Supply chain management survey of Swedish manufacturing firms. International Journal of Production Economics, 89 (2004), 353-361.

[11] E.L. Porteus: Optimal lot sizing, process quality improvement and setup cost reduction. Operations Research, 34 (1986), 137-144.

[12] T. Raz, Y.T. Herer and A. Grosfeld-Nir: Economic optimization of off-line inspection. IIE Transaction, 32 (2000), 205-217.

[13] H. Stackelberg: The Theory of the Market Economy (Oxford University Press, New York, 1952).

[14] D. Simchi-Levi, P. Kaminsky and E. Simchi-Levi: Design and managing the supply chain (McGraw-Hill Irwin, Inc., New York,1996).

[15] C.S. Tapiero: Yield and control in a supplier-customer relationship. International Journal of Production Research, 39 (2001), 1505-1515.

[16] S.H. Tseng: Optimal preventive maintenance policy for deteriorating production systems. IIE transactions, 28-8 (1996), 687-694.

[17] C.H. Wang and S.H. Sheu: Optimal lot sizing for products sold under free-repair warranty. European Journal of Operational Research, 149 (2003), 131-141.

[18] C.H. Wang: Integrated production and product inspection policy for a deteriorating production system. International Journal of Production Economics, 95 (2005), 123134.

[19] R.H. Yeh, W.T. Ho and S.T. Tseng: Optimal production run length for products sold with warranty. European Journal of Operational Research, 120 (2000), 575-582.

Chih-Hsiung Wang

National Pingtung Institute of Commerce

51 Min-Sheng E. Road, Pingtung 900, Taiwan

E-mail: chwang@npic.edu.tw 